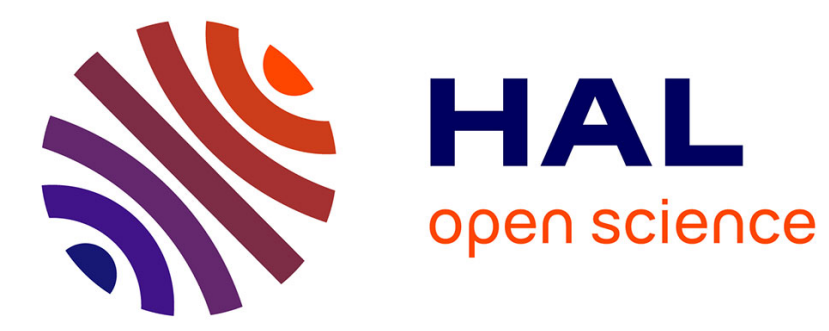

\title{
Uplink capacity of self-organizing clustered orthogonal CDMA networks in flat fading channels
}

\author{
Romain Couillet, Merouane Debbah
}

\section{To cite this version:}

Romain Couillet, Merouane Debbah. Uplink capacity of self-organizing clustered orthogonal CDMA networks in flat fading channels. ITW-2009, Oct 2009, Italy. 5 p. hal-00414531

HAL Id: hal-00414531

https://hal-centralesupelec.archives-ouvertes.fr/hal-00414531

Submitted on 13 Jan 2010

HAL is a multi-disciplinary open access archive for the deposit and dissemination of scientific research documents, whether they are published or not. The documents may come from teaching and research institutions in France or abroad, or from public or private research centers.
L'archive ouverte pluridisciplinaire HAL, est destinée au dépôt et à la diffusion de documents scientifiques de niveau recherche, publiés ou non, émanant des établissements d'enseignement et de recherche français ou étrangers, des laboratoires publics ou privés. 


\section{Uplink capacity of self-organizing clustered orthogonal CDMA networks in flat fading channels}

\author{
Romain Couillet \\ ST-Ericsson, Supelec \\ 505 Route des Lucioles \\ 06560 Sophia Antipolis, France \\ Email: romain.couillet@supelec.fr
}

\author{
Mérouane Debbah \\ Alcatel-Lucent Chair, Supelec \\ Plateau de Moulon, 3 rue Joliot-Curie \\ 91192 Gif sur Yvette, France \\ Email: merouane.debbah@supelec.fr
}

\begin{abstract}
In this paper, we derive a deterministic equivalent of the Shannon-transform of certain type of large unitary random matrices. This approximation is exploited to evaluate the uplink channel capacity of clustered orthogonal CDMA network. When non-uniform power allocation among the users of each cluster is allowed, we derive an explicit iterative waterfilling algorithm which, upon convergence, achieves the multiuser decoding capacity. In particular, we show that, in a selforganizing clustered orthogonal CDMA network, each cluster can optimize its power allocation policy independently of the other clusters at the expense of a small feedback overhead. Simulations corroborate the theoretical derivations.
\end{abstract}

\section{INTRODUCTION}

Previous releases of the universal mobile telecommunications system (UMTS) aimed to ensure quality of service for all users in the cells. The recent developments of $3 \mathrm{G}$ protocols however push more and more towards the maximization of the user sum rates in the cell. 3G systems are based on code division multiple access (CDMA) which use random i.i.d. codes in the uplink and incur interference among the users. Orthogonal codes (which reduce drastically the interference) are unfortunately not used as it would require some important overhead signaling to synchronize the users. One way of dealing with this issue, which is part of the recent cognitive radio incentive [2], proposes to allow users to self-organize in small clusters (e.g. here CDMA-based clusters) or coalitions [11], [12] to optimize their uplink communications to a central base station [13], [14]. Due to the very short distance between the users within the cluster, non-interfering transmissions can be performed through the use of orthogonal codes per cluster. Those networks are referred to as self-organizing networks, which we shall consider in the following.

Among the past contributions in the multi-cell CDMA case, a performance study of multi-cell orthogonal CDMA networks in frequency selective channels was carried out in [3]. The authors suggest that frequency selectivity has a stronger negative impact on CDMA performance than the path loss; therefore multiple small CDMA cells might turn out more advantageous than a large single CDMA cell. The same conclusions were given in a similar uplink random CDMA study in [4]. In [5], the performance of single-cell CDMA in flat-fading channels with random codes was analyzed for different types of decoders when power optimization is performed at the transmitter. Most of these studies derive results under the assumption that the number of users per cell is large, since simpler derivations are possible using the theory of large random matrices [6].

In this paper we derive results for the uplink capacity of a clustered CDMA self-organizing network when the CDMA code matrices are large and isometric. ${ }^{1}$ Those results are based on tools from random matrix theory [6] and free probability [7], [8]. We will in particular derive two theorems which relate respectively the $\eta$-transform and the Shannon-transform (see definitions hereafter) of such a channel model to deterministic approximations independent of the code matrix realization. Another deterministic equivalent of the $\eta$-transform has been considered recently in the frequency selective case by Peacock et al. [1] using a different approach, referred to as "incremental matrix expansions". However, the form of their deterministic approximations does not allow for a trivial calculus of the Shannon-transform, which is provided here. Up to the authors' understanding, both our and Peacock's results are different, though they are shown to tend to the same asymptotic expression. Our result on the Shannon-transform allows us first to derive explicit expressions for the uplink clustered orthogonal CDMA capacity and more importantly to produce an iterative water-filling algorithm for the cell users to achieve network capacity under per-cluster sum power constraint (where the power within a cluster is seen as a long term exchangeable utility, see [12] for more justifications). We will also propose an algorithm for each cluster to automatically reconfigure their power allocations, independently of the other clusters, when a user connects or disconnects to the cell.

The remainder of this paper unfolds as follows: in Section II, the fundamental mathematical results of this paper are provided. In Section III, the clustered CDMA self-organizing network model is introduced. In Section IV, the capacity achieving power allocation algorithm is presented. In Section $\mathrm{V}$, numerical simulations are carried out. Finally, in Section VI, we draw our conclusions.

Notation: Capital boldface characters denote matrices $\left(\mathbf{I}_{N}\right.$

${ }^{1}$ by isometric, we mean a non-necessarily square matrix with orthogonal columns. 
is the $N \times N$ identity matrix). Hermitian transpose is denoted $(\cdot)^{\mathrm{H}}$. The operator det $\mathbf{X}$ represents the determinant of the matrix $\mathbf{X}$. The function $\delta(x)$ is the Kronecker Dirac function such that $\delta(x)=1$ if $x=0$ and equals 0 otherwise. The first derivative of the function $f$ is denoted $f^{\prime}$.

\section{Mathematical Preliminaries}

Our main results rely on tools of random matrix theory analysis, the $\eta$-transform and the Shannon-transform, which we introduce in the following.

Definition 1 ( $\eta$-transform): Let $\mu$ be a probability density function. The $\eta$-transform $\eta(x)$ of $\mu$ is defined as

$$
\eta(x)=\int \frac{1}{1+x t} \mu(d t)
$$

Note that the $\eta$-transform is linked to the better known Stieltjes transform $S(x)$ via the relation $S(-1 / x)=x \eta(x)$.

Theorem 1: Let $K, N$ be positive integers, $\left\{\mathbf{W}_{i}\right\}_{i=1, \ldots, K}$ be $K$ independent $N \times N$ Haar distributed complex random matrices and $\left\{\mathbf{D}_{i}\right\}_{i=1, \ldots, K}$ be $K$ diagonal $N \times N$ non-negative matrices. Denote $\mu_{i}$ the empirical distribution of the entries of $\mathbf{D}_{i}$. Then, for $N$ large, $K$ fixed, and for some $x \geq 0$, the $\eta$-transform $\eta(x)$ of the empirical eigenvalue distribution of $\mathbf{B}_{N}=\sum_{k=1}^{K} \mathbf{W}_{k} \mathbf{D}_{k} \mathbf{W}_{k}^{\mathrm{H}}$ satisfies approximately

$$
\eta(x)=\left(1+x \sum_{k=1}^{K} \beta_{k}(x)\right)^{-1}
$$

where the functions $\beta_{k}(x), k \in\{1, \ldots, K\}$ satisfy the $K$ fixed-point equations

$$
\beta_{k}(x)=\int \frac{t}{1-x \eta(x) \beta_{k}(x)+x \eta(x) t} \mu_{i}(d t)
$$

Proof: The proof relies mostly on the asymptotic freeness [6] of the matrices $\mathbf{W}_{k} \mathbf{D}_{k} \mathbf{W}_{k}^{\mathrm{H}}$ when $N$ grows to infinity. In this case, the $R$-transform $R(x)$ of $\mathbf{B}_{N}$ is asymptotically equal to the sum of the $R$-transform $R_{k}(x)$ of $\mathbf{W}_{k} \mathbf{D}_{k} \mathbf{W}_{k}^{\mathrm{H}}$. This $R$ transform is defined with respect to the $\eta$-transform as ${ }^{2}$

$$
\begin{aligned}
R(-x \eta) & =-\frac{1}{x}\left(1-\frac{1}{\eta}\right) \\
\eta\left(-\frac{1}{R+\frac{1}{x}}\right) & =x R+1
\end{aligned}
$$

Denote $\eta_{k}$ and $R_{k}$ the $\eta$ - and $R$-transform of $\mathbf{W}_{k} \mathbf{D}_{k} \mathbf{W}_{k}^{\mathrm{H}}$. Then, from (1) and (5), we have

$$
x R_{k}+1=\int \frac{1}{1-\frac{t}{R_{k}+\frac{1}{x}}} \mu_{k}(d t)
$$

which leads to

$$
R_{k}(x)=\frac{1}{x} \int \frac{t}{R_{k}(x)+\frac{1}{x}-t} \mu_{k}(d t)
$$

and, in particular, defining $\beta_{k}(x)=R_{k}(-x \eta)$, we have

$$
\beta_{k}(x)=\int \frac{t}{1-x \eta \beta_{k}+x \eta t} \mu_{k}(d t)
$$

\footnotetext{
${ }^{2}$ for readability, we will use the shortcut notation $f=f(x)$ when no confusion is possible.
}

which is exactly (3).

Now, since $R=\sum_{k=1}^{K} R_{k}$, using (4), this gives

$\eta(x)=\left(1+x \sum_{k=1}^{K} R_{k}(-x \eta)\right)^{-1}=\left(1+x \sum_{k=1}^{K} \beta_{k}\right)^{-1}$

which completes the proof.

The $\eta$-transform is of no direct practical use in our current analysis (though it might be essential in the analysis of MMSE decoders for CDMA downlink, see e.g. [1]). To establish the capacity of uplink transmissions in CDMA networks, we need a deterministic approximation of the so-called Shannontransform, defined as follows.

Definition 2 (Shannon-transform): Let $\mu$ be some probability density function. We define the Shannon-transform $\mathcal{V}(x)$ of $\mu$ as

$$
\mathcal{V}(x)=\int \log (1+x t) \mu(d t)
$$

The Shannon-transform is intimately linked to the capacity of a multi-dimensional channel whose matrix empirical eigenvalue density function is $\mu$. Note also that the Shannon-transform is an integral of $1 / x(1-\eta(x))$. From this observation, equivalently to Theorem 1 , we derive in the following theorem a deterministic approximation for $\mathcal{V}(x)$.

Theorem 2: Let $\mathbf{B}_{N}$ be an $N \times N$ matrix as defined in the conditions of Theorem 1. We have the following asymptotic approximation of the Shannon-transform $\mathcal{V}(x)$ of $\mathbf{B}_{N}$ as

$$
\begin{aligned}
\mathcal{V}(x)= & \log \left(1+x \sum_{k=1}^{K} \beta_{k}(x)\right) \\
& +\sum_{k=1}^{K} \int \log \left(1+x \eta(x)\left[t-\beta_{k}(x)\right]\right) \mu_{k}(d t)
\end{aligned}
$$

where $\beta_{k}(x)$ and $\eta(x)$ are given by (2) and (3).

Proof: The Shannon-transform satisfies [6]

$$
\mathcal{V}(x)=\int_{0}^{x} \frac{1}{u}(1-\eta(u)) d u
$$

we therefore seek an integral form for the $\eta$-transform. Note first that

$$
\frac{1}{x}(1-\eta)=\frac{1}{x}\left(1-\left(1+x \sum_{k=1}^{K} \beta_{k}\right)^{-1}\right)=\sum_{k=1}^{K} \beta_{k} \eta
$$

Also note from (3) that

$$
1-x \eta \beta_{k}=\int \frac{1-x \eta \beta_{k}}{1-x \eta \beta_{k}+x \eta t} \mu_{k}(d t)
$$

and therefore that

$$
1=\int \frac{1}{1-x \eta \beta_{k}+x \eta t} \mu_{k}(d t)
$$

Now, for any $k$, the derivative along $x$ of $C_{k}=\int \log (1-$ $\left.x \eta \beta_{k}+x \eta t\right) \mu_{k}(d t)$ is

$$
C_{k}^{\prime}=\int \frac{\left[-\eta \beta_{k}-x \eta^{\prime} \beta_{k}-x \eta \beta_{k}^{\prime}\right]+\left[\eta+x \eta^{\prime}\right] t}{1-x \eta \beta_{k}+x \eta t} \mu_{k}(d t)
$$




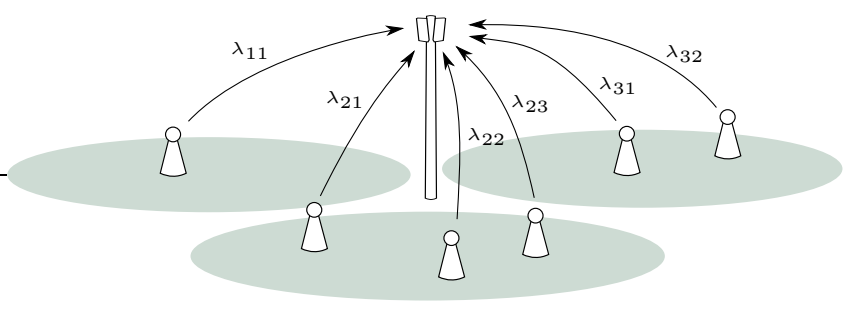

Fig. 1. Self-organizing clustered CDMA network

Recalling now (13) and (3), this yields

$$
C_{k}^{\prime}=\left(-\eta \beta_{k}-x \eta^{\prime} \beta_{k}-x \eta \beta_{k}^{\prime}\right) \cdot 1+\left(\eta+x \eta^{\prime}\right) \cdot \beta_{k}=-x \eta \beta_{k}^{\prime}
$$

We also have

$$
\left(\log \left(1+x \sum_{k=1}^{K} \beta_{k}\right)\right)^{\prime}=\eta \sum_{k=1}^{K} \beta_{k}+x \sum_{k=1}^{K} \eta \beta_{k}^{\prime}
$$

Adding this last expression to $\sum_{k} C_{k}^{\prime}$, we end up with the desired $\sum_{k=1}^{K} \beta_{k} \eta$. Verifying that $\mathcal{V}(0)=0$, we finally obtain (10).

\section{SySTEM MODEL}

\section{A. Model}

Consider a network composed of $K$ clusters $^{3}$ and a single base station, each cluster being composed of a maximum of $N$ users each. The users of cluster $k \in\{1, \ldots, K\}$ are assigned orthogonal spreading codes forming the columns of a unitary $N \times N$ Haar distributed matrix $\mathbf{W}_{k}$. Each cluster operates independently regarding the allocation of spreading codes, and therefore the $\mathbf{W}_{k}$ matrices are considered independent. Depending on its position in cluster $k$, user $n \in\{1, \ldots, N\}$ induces a path-loss $\lambda_{k n}$ in the uplink transmission to the base station ${ }^{4}$. The path-losses in cluster $k$ are gathered in the diagonal matrix $\boldsymbol{\Lambda}_{k}=\operatorname{diag}\left(\lambda_{k 1}, \ldots, \lambda_{k N}\right)$. Moreover we assume that the transmission channel is frequency flat, i.e. the propagation channel fade is constant over the transmission bandwidth. User $n$ in cluster $k$ transmits with power $p_{k n}$, the collection of which is gathered in the matrices $\mathbf{P}_{k}=$ $\operatorname{diag}\left(p_{k 1}, \ldots, p_{k N}\right)$. We additionally assume the base station experiences a background noise distributed as $\operatorname{C\mathcal {N}}\left(0, \sigma^{2}\right)$. This situation is depicted in Figure 1.

\section{B. Uplink capacity}

Denoting $\mathbf{D}_{k}=\boldsymbol{\Lambda}_{k} \mathbf{P}_{k}$, the uplink (per-time sample) capacity $C$ reads

$$
C\left(\sigma^{2}\right)=\frac{1}{N} \log \operatorname{det}\left(\mathbf{I}_{N}+\frac{1}{\sigma^{2}} \sum_{k=1}^{K} \mathbf{W}_{k} \mathbf{D}_{k} \mathbf{W}_{k}^{\mathrm{H}}\right)
$$

\footnotetext{
${ }^{3}$ we assume that the clusters are formed in a self-organized manner based on the distance, see [12]

${ }^{4}$ when there are less than $N$ users in a specific cluster, we can freely write $\lambda_{k n}=0$ for the virtual users.
}

Denoting $\mu_{k}(t)=\frac{1}{N} \sum_{n=1}^{N} \delta\left(t-\lambda_{k n} p_{k n}\right)$, from Theorem 2 , when $N$ is large, we have approximately ${ }^{5}$

$$
\begin{aligned}
C\left(\sigma^{2}\right) & =\log \left(1+\frac{1}{\sigma^{2}} \sum_{k=1}^{K} \beta_{k}\left(\sigma^{-2}\right)\right) \\
& +\sum_{k=1}^{K} \int \log \left(1+\sigma^{-2} \eta\left(\sigma^{-2}\right)\left[t-\beta_{k}\left(\sigma^{-2}\right)\right]\right) \mu_{k}(d t)
\end{aligned}
$$

with $\eta(x)$ and $\beta_{k}(x)$ satisfying the implicit Equation (3). We therefore have an expression of the uplink capacity which only depends on the path losses $\lambda_{k n}, k \in\{1, \ldots, K\}$, $n \in\{1, \ldots, N\}$. This suggests that the choice of the spreading codes $\mathbf{W}_{k}$ 's has asymptotically no impact on the achievable uplink sum rate. We will now seek a power allocation strategy which maximizes the capacity under cluster power constraints.

\section{SELF-ORGANIZED POWER ALLOCATION}

In the following, based on relative fairness among clusters and on individual mobile terminal capabilities, cluster $k$ is allocated a total allowed transmit $P_{k}$; therefore $\sum_{n=1}^{N} p_{k n}=$ $P_{k}$.

\section{A. Optimal power allocation}

To maximize the system capacity with respect to the $K N$ scalars $p_{k n}$, we shall first show the following lemma,

Lemma 1: The power allocation policy $p_{k n}=p_{k n}^{\star}$ optimizing the deterministic capacity approximation (18) satisfies, for all $k, n$,

$$
p_{k n}^{\star}=\left(\alpha_{k}-\frac{\sigma^{2}-\eta^{\star} \beta_{k}^{\star}}{\lambda_{k n} \eta^{\star}}\right)^{+}
$$

where $\eta^{\star}, \beta_{k}^{\star}$ are the respective values of $\eta$ and $\beta_{k}$ when $C$ achieves its maximum, and $\alpha_{k}$ is such that $\sum_{k} p_{k n}^{\star}=P_{k}$.

Proof: To prove this lemma, we follow Prop. 4 in [10]. We need to ensure successively that, denoting $C\left(\sigma^{2}\right)=$ $V\left(p_{11}, \ldots, p_{K N}, \beta_{1}, \ldots, \beta_{K}, \eta\right){ }^{6}$

(i) $\partial V / \partial \beta_{k}=\partial V / \partial \eta=0$.

(ii) $C$ is strictly concave in the $p_{k n}$.

Part (ii) is in fact obvious once (i) is shown. Indeed, in this case, the successive derivatives of $C$ along $p_{k n}$ equal those of $V$ along $p_{k n}$ plus other terms (the derivatives of $V$ along $\eta$ and $\beta_{k}$ ) which are null. Therefore, since $V$ is the sum of strictly concave functions of the $p_{k n}, C$ is strictly concave.

To show (i), we simply differentiate and observe that

$$
\begin{aligned}
& \frac{\partial V}{\partial \eta}=\sum_{k} \int \frac{t \sigma^{-2}-\beta_{k} \sigma^{-2}}{1-\eta \beta_{k} \sigma^{-2}+\eta \sigma^{-2} t} \mu_{k}(d t)=0 \\
& \frac{\partial V}{\partial \beta_{k}}=\eta \sigma^{-2}+\int \frac{-\eta \sigma^{-2}}{1-\eta \beta_{k} \sigma^{-2}+\eta \sigma^{-2} t} \mu_{k}(d t)=0
\end{aligned}
$$

both equalities stemming from the remarks (13) and (3).

\footnotetext{
${ }^{5}$ note that the capacity is the Shannon-transform of $\sigma^{-2}$ and not of $\sigma^{2}$.

${ }^{6} \mathrm{~V}$ can be seen as a function of $(N+1) K+1$ independent variables.
} 
From (ii), $C$ admits a unique maximum and from (i), when $\beta_{k}$ and $\eta$ have reached this maximum, the power allocation of the $p_{k n}$ 's reduces to maximizing

$\log \left(1+\sigma^{-2} \sum_{k=1}^{K} \beta_{k}\right)+\sum_{k=1}^{K} \int \log \left(1+\sigma^{-2} \eta\left[t-\beta_{k}\right]\right) \mu_{k}(d t)$

independently of $\eta$ and the $\beta_{k}$ 's. This is, for each $k$, it reduces to maximizing $\int \log \left(1+\sigma^{-2} \eta\left[t-\beta_{k}\right]\right) \mu_{k}(d t)$, whose solution is the water-filling solution (19).

To achieve the capacity optimizing power allocation policy, similarly to [10], we propose the following iterative waterfilling algorithm,

At initialization, for all $k, p_{k n}=\frac{P_{k}}{N}, \eta=1, \beta_{k}=1$.

while the $p_{k n}$ 's have not converged do

for $k \in\{1, \ldots, K\}$ do

Set $\left(\eta, \beta_{k}\right)$ as solution of (2), (3)

for $n=1 \ldots, N$ do

Set $p_{k n}=\left(\alpha_{k}-\frac{\sigma^{2}-\eta \beta_{k}}{\lambda_{k n} \eta}\right)^{+}$, with $\alpha_{k}$ such that $\sum_{n} p_{k n}=P_{k}$.

$$
\text { end for }
$$

end for

end while

This algorithm cannot be proved to converge. However, from the proof of Lemma 1 and following the arguments in [10], upon convergence, the algorithm is proved to converge to the optimal solution.

The power allocation algorithm allows now the base station to indicate to all users the uplink transmission powers, provided that it is aware of all the $\lambda_{k n}$ 's. We will show in the following that such a centralized approach is unnecessary to achieve optimal power allocation; precisely, we will show that each cluster $k$ can independently perform power allocation regardless of the $\lambda_{j n}, j \neq k$, provided that each cluster feedbacks a single real parameter to a neighboring cluster after the individual optimization process.

\section{B. Self-organized capacity optimization}

The main interest of the system model under consideration lies in the independence of every cluster in terms of code allocation: each cluster can behave autonomously, without the need for the base station to intervene in the code allocation policy. Now, in order to achieve the optimal uplink capacity throughout the $K$ clusters, user $n$ in cluster $k$ must be allocated a transmit power $p_{k n}=p_{k n}^{\star}$. Those $p_{k n}$ 's can be computed explicitly at the base station or by any central entity aware of all the $\lambda_{k n}$ 's. However, this requires a large amount of overhead data to be transferred back and forth through the network; this comes at a non-negligible cost and goes against the philosophy of self-organized networks.

It is actually possible to circumvent this issue by performing successive local optimizations in every cluster and by successively transmitting a single parameter to the other clusters: this parameter is the updated evaluation of the $\eta$ transform of $\mathbf{B}_{N}$. More explicitly, we consider the following algorithm,

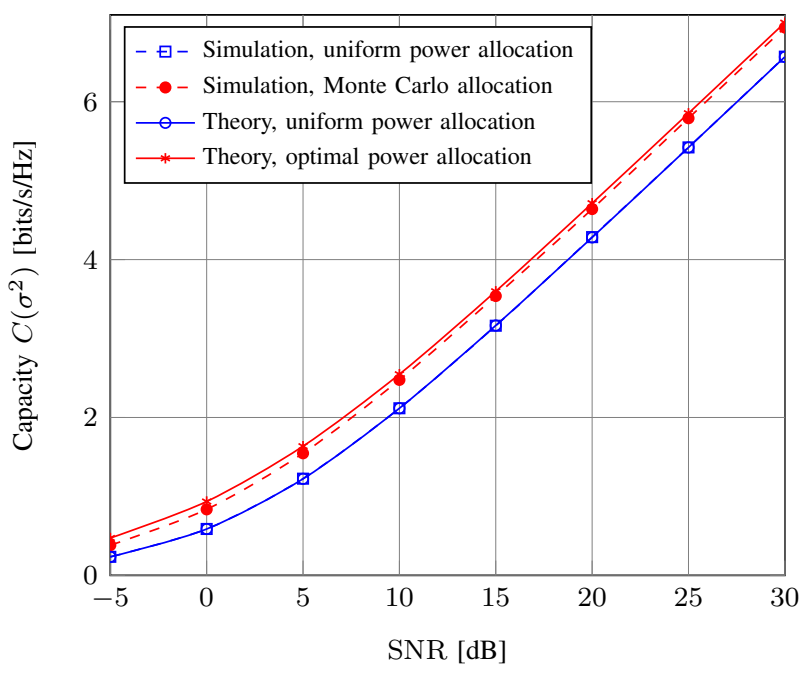

Fig. 2. Uplink capacity of a $K=3$-clustered orthogonal CDMA, uniform and optimal power allocation, $N=8$. For user $n$ in cluster $k, \lambda_{k n}=$ $-\alpha_{k}([n-1] /[N-1]) \mathrm{dB}, \alpha_{1}=10 \mathrm{~dB}, \alpha_{2}=20 \mathrm{~dB}, \alpha_{3}=30 \mathrm{~dB}$.

At initialization, in cluster $k$, set $p_{k n}=\frac{P_{k}}{N}, \eta=1$ and $\beta_{k}=1$.

while $\eta$ has not converged do

for cluster $k \in\{1, \ldots, K\}$ do

Compute $\eta$ as in (2).

Set $\beta_{k}$ as solution of (3), from the updated $\eta$.

for $n=1 \ldots, N$ do

Set $p_{k n}=\left(\alpha_{k}-\frac{\sigma^{2}-\eta \beta_{k}}{\lambda_{k n} \eta}\right)^{+}$, with $\alpha_{k}$ such that $\sum_{n} p_{k n}=P_{k}$.

end for

Transmit the updated value $\eta$ to cluster $k+1$ (with convention $1 \leftarrow K+1$ ).

\section{end for}

end while

This algorithm only requires for the clusters to transmit the updated value of $\eta$ at each step. Note that computing $\eta$ requires only to know the updated value of $\sum_{k} \beta_{k}$. This value does not need be additionally transmitted if we assume that each cluster stores the last value of $\eta$ and infers from it the updated $\sum_{k} \beta_{k}$.

This way, when a new user registers or a current user disconnects from a cluster, all the transmitted powers can be quickly updated throughout the network at a low feedback cost. Moreover, as will be observed in Section V, the convergence time of the algorithm is in general so fast that a single round of optimization over the $K$ clusters is sufficient (and therefore the outer while loop is not needed) to achieve a satisfying uplink rate.

In the next section, the theoretical results discussed so far are confronted to simulations.

\section{Simulation and Results}

In this section, we verify the theoretical formula derived in Theorem 2, the power allocation algorithm derived from Lemma 1 and the self-organizing successive power allocation proposed in Section IV-B. Throughout this section, we 


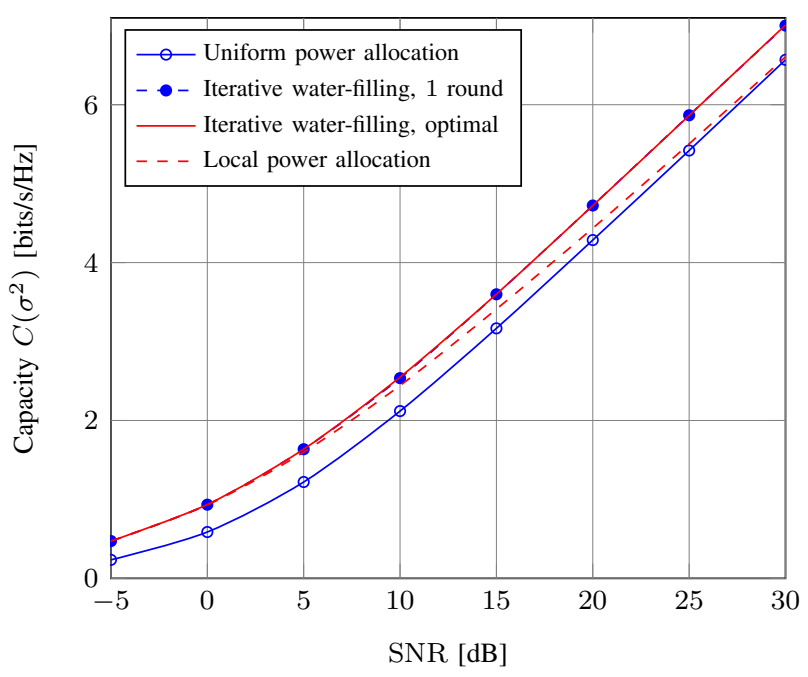

Fig. 3. Uplink capacity of a $K=3$-cluster orthogonal CDMA, iterative water-filling power allocation, $N=8$. For user $n$ in cluster $k, \lambda_{k n}=$ $-\alpha_{k}([n-1] /[N-1]) \mathrm{dB}, \alpha_{1}=10 \mathrm{~dB}, \alpha_{2}=20 \mathrm{~dB}, \alpha_{3}=30 \mathrm{~dB}$.

consider a $K=3$-cluster self-organizing orthogonal CDMA network with $N=8$ users per cluster. The uplink signal from user $n$ in cluster $k$ experiences a path loss $\lambda_{k n}=$ $-\alpha_{k}([n-1] /[N-1]) \mathrm{dB}$, with $\alpha_{1}=10 \mathrm{~dB}, \alpha_{2}=20 \mathrm{~dB}$, $\alpha_{3}=30 \mathrm{~dB}$; this allows to have in each cluster a linear range (in $\mathrm{dB}$ ) of users with relative path losses varying from $0 \mathrm{~dB}$ to $\left(-\alpha_{k}\right) \mathrm{dB}$.

In Figure 2, we present simulation results based on the averaging of 100 Monte Carlo simulations when uniform power allocation is applied or when the allocated powers are issued from random Monte Carlo trials (but satisfy the power constraints); for the latter, for each of the 100 simulations, we define the system capacity as the maximal rate achieved over 10,000 random realizations of vectors $\left\{p_{11}, \ldots, p_{K N}\right\}$ ordered in such a way that $p_{k j}<p_{k, j+1} .{ }^{7}$ Those are compared against the solutions given by Theorem 2 with uniform power allocation and the iterative water-filling algorithm, respectively. We observe a perfect fit, even for this low $N=8$ value, between the simulated and theoretical results in the uniform power allocation case. As for the optimal power allocation, we observe an almost perfect fit, which will surely be more accurate if one increases the number of random power allocations of the Monte Carlo method.

In Figure 3, we apply the self-organizing power allocation algorithm under the same constraints as in Figure 3, when 0, 1 or an infinite number of rounds (at least a sufficient number of rounds to ensure convergence of $\eta$ ) are performed). We observe surprisingly enough that a single round is enough to already achieve the optimal capacity limit of the complete algorithm. As a consequence, the algorithm is extremely fast at providing high performance power allocation among the $K$ clusters. For the sake of comparison, we also propose in Figure 3 the so-called 'local power allocation' policy applied

\footnotetext{
${ }^{7}$ this allows to significantly reduce the number of required simulations by discarding obviously wrong solutions.
}

by each cluster as though it were alone in the network, which is maximized by the classical water-filling solution, i.e. with powers

$$
p_{k n}=\left(\alpha_{k}^{\prime}-\frac{\sigma^{2}}{\lambda_{k n}}\right)^{+}
$$

where $\alpha_{k}^{\prime}$ ensures that $\sum_{n} p_{k n}=P_{k}$. We observe that, while in the low SNR region, this local strategy is reasonable, it is no longer the case in the high SNR regime. Since the cost of the transmission of the datum $\eta$ is obviously very low in this regime, the self-organizing power allocation policy is even more interesting.

\section{CONCLUSION}

In this paper, we provided two novel theorems relating the uplink capacity of a large clustered orthogonal frequency flat CDMA network communicating to a single base station to deterministic capacity approximations. Those actually very accurate approximations only depend on the path losses of the users' uplink signals. Moreover, we provided an efficient algorithm for the clusters to perform local power allocation in order to maximize the system throughput at the cost of a single datum exchange in the network. Simulation results show a perfect fit between Monte Carlo and theoretical results even when the system dimension is not very large.

\section{REFERENCES}

[1] M. J. M. Peacock, I. B. Collings and M. L. Honig, "Eigenvalue distributions of sums and products of large random matrices via incremental matrix expansions," IEEE Trans. on Information Theory, vol. 54, no. 5, pp. 2123, 2008.

[2] J. Mitola III and G. Q. Maguire Jr, "Cognitive radio: making software radios more personal," IEEE personal communications, vol. 6, no. 4, pp. 13-18, 1999.

[3] M. Debbah, "Downlink CDMA: to cell or not to cell," 12th European Signal Processing Conference, Vienna, Austria, 2004

[4] O. Somekh, B.J. Zaidel and S. Shamai, "Spectral Efficiency of Joint Multiple Cell-Sites Processors for Randomly Spread DS-CDMA,” ISIT, Chicago, USA, 2004.

[5] S. Shamai and S. Verdu, "The Impact of Frequency-Flat Fading on the Spectral Efficiency of CDMA," IEEE Trans. on Information Theory, pp. 1302-1326, May 2001.

[6] A. Tulino, S. Verdú, "Random Matrix Theory and Wireless Communications," Now Publishers, vol. 1, issue 1, 2004

[7] D.V. Voiculescu, K.J. Dykema and A. Nica "Free random variables," American Mathematical Society, 1992.

[8] P. Biane, "Free probability for probabilists," High Magnetic Fields: Science and Technology, World Scientific, pp. 55, 2003.

[9] T.M. Cover, and J.A. Thomas, "Elements of information theory," Wiley New York, 1991.

[10] J. Dumont, S. Lasaulce, W. Hachem, Ph. Loubaton and J. Najim, "On the Capacity Achieving Covariance Matrix for Rician MIMO Channels: An Asymptotic Approach", submitted to IEEE Trans. on Information Theory, October 2007, revised in October 2008.

[11] A. Coso, S. Savazzi, U. Spagnolini, and C. Ibars, "Virtual MIMO channels in cooperative multi-hop wireless sensor networks", in Proc. Conf. on Information Sciences and Systems, Mar. 2006, pp. 75-80

[12] W. Saad, Z. Han, M. Debbah, and A. Hjørungnes, "A distributed merge and split algorithm for fair cooperation in wireless networks," in Proc. Int. Conf. on Communications, Workshop on Cooperative Communications and Networking, Beijing, China, 2008.

[13] N. Jindal, U. Mitra, and A. Goldsmith, "Capacity of ad-hoc networks with node cooperation," in Proc. Int. Symp. on Information Theory, Chicago, IL, USA, Jun. 2004, pp. 271.

[14] J. Cai and U. Pooch, "Allocate fair payoff for cooperation in wireless ad hoc networks using shapley value," in Proc. Int. Parallel and Distributed Processing Symposium, Santa Fe, NM, USA, Apr. 2004, pp. 219227. 